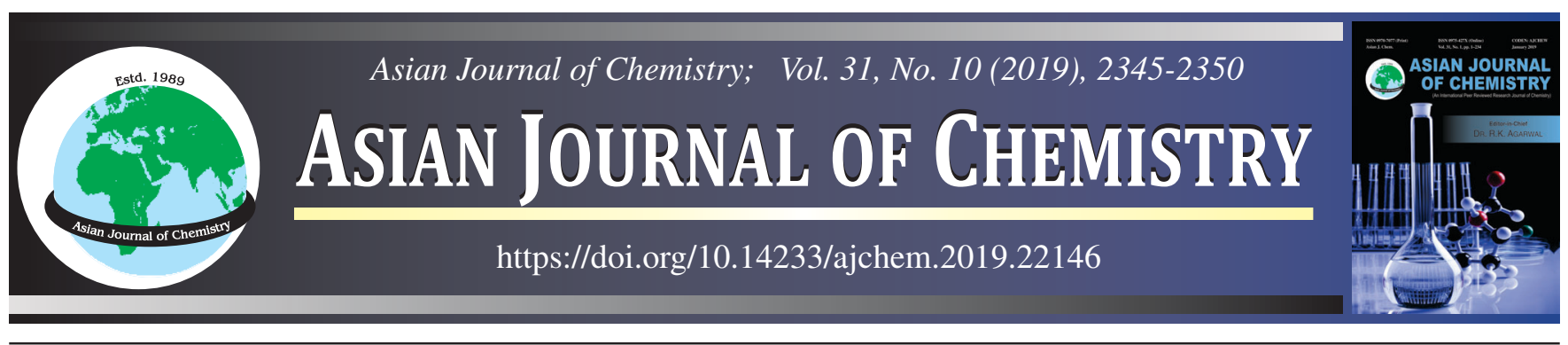

\title{
Interactions of Xanthan Gum and Carboxymethyl Cellulose on Physical and Sensory of Cloudy Asparagus Juice using Response Surface Methodology
}

\author{
Nguyen Thi Van Linh ${ }^{1, *}$, Vung Thi Ngoc Mai ${ }^{1}$, Tran Thi Yen NhI ${ }^{2,3}$ and Tri Duc Lam ${ }^{2,3}$
}

${ }^{1}$ Faculty of Chemical Engineering and Food Technology, Nguyen Tat Thanh University, Ho Chi Minh City, Vietnam

${ }^{2}$ NTT Hi-Tech Institute, Nguyen Tat Thanh University, Ho Chi Minh City, Vietnam

${ }^{3}$ Center of Excellence for Biochemistry and Natural Products, Nguyen Tat Thanh University, Ho Chi Minh City, Vietnam

*Corresponding author: E-mail: ntvlinh@ntt.edu.vn

Received: 23 April 2019;

Accepted: 17 June 2019;

Published online: 30 August 2019;

AJC-19549

This study aims to investigate the effect of xanthan gum and carboxymethylcellulose on physical and chemical qualities of green asparagus juice. We adopted the surface-response method and the CCD experiment design with respect to three response variables including stability, viscosity and colour of the product. It was revealed that both xanthan gum and carboxymethylcellulose concentrations are both positively correlated with viscosity and stability of the product. In addition, the coefficient of the interaction of xanthan gum and carboxymethylcellulose was not significant $(\mathrm{p}<0.05)$. In sensory evaluation, both carboxymethylcellulose and xanthan gum were found to be influential on product state. However, hydrocolloid concentration effects were not profound on perceived product colour and odour.

Keywords: Xanthan gum, Asparagus, Hydrocolloids, Carboxymethyl cellulose, Response surface methodology

\section{INTRODUCTION}

Nowadays, using natural products have been receiving a great deal of public attention [1-3]. The plant organs such as seeds, flowers, roots are applied in different fields and purposes including the manufacture of antimicrobial compounds, medicine and food additives [4,5]. Asparagus is a perennial plant of the liliaceous family, and asparagus shoots are used in meals such as salads, soups and vegetables throughout the world. It is also used as a traditional herbal medicine in Europe and Asia [6-8]. In particular, asparagus shoots are rich in biologically active substances reported to have antioxidant, anticancer and anti-inflammatory activities. Saponin steroids are a component found in asparagus with potent anticancer activity $[9,10]$.

Moreover, asparagus is a healthy and nutritious food that has many biologically active phytochemicals such as carotenoids, flavonoids, phenols, fiber and oligosaccharides. In addition to the culinary quality, asparagus and asparagus extracts are reported to have antioxidant, antitumour, hypolipidaemic, hypoglycaemic and antifungal properties [11]. Human diet plays a crucial role in protecting against oxidative stress.
Asparagus extracts have antiaging effects by improving insulin secretion and cellular function (sup) and antioxidant status [12]. Fresh asparagus is preserved for up to 7 days at $3{ }^{\circ} \mathrm{C}$, but the asparagus tops are the most perishable organ. The fresh asparagus is preserved by either rapid cooling by water or lignification [13]. However, asparagus is still difficult to be preserved for a long time. Therefore, the products originating from the environment such as asparagus nectar, asparagus juice, asparagus powder, asparagus tea, asparagus have been studied. Fruit juices are a product that consumers care about for the health benefits and nutritional value it provides. These fruit juices are also described to have a difference in therapeutic properties in some physiological conditions for the recovery of the human body. In Nagpur city of India, fresh fruit and vegetable consumption is increasing throughout the year because fresh fruit and vegetable juices are highly favoured for their healthy health effects [14]. In Turkey, pomegranate (Punica granatum) is consumed as a fruit and fruit juice with high antioxidant activity and also used in salads and desserts $[15,16]$. Beside colour or taste, consumers are concerned about the sedimentation, layer separation or if there is a lipid layer on the top of not all are considered as a defect for the product. Consumers are concerned about the

This is an open access journal, and articles are distributed under the terms of the Attribution 4.0 International (CC BY 4.0) License. This license lets others distribute, remix, tweak, and build upon your work, even commercially, as long as they credit the author for the original creation. You must give appropriate credit, provide a link to the license, and indicate if changes were made. 
stability of the product whether it is fresh or powdered, turbid juice products from fruits such as oranges, tangerines, coconuts which are normally encountered in the processing progress [17]. The deposition rate depends on the diameter of the grain, the difference in the density of the liquid phase and the solid phase, the viscosity [18].

In order to limit the layer separation of lentil nectar, the addition of xanthan gum, guar gum and carrageenan to limit sedimentation and stability to nectar [19]. In one study of apple juice production, carboxymethylcellulose, xanthan gum was added to keep the product stable [20]. For carrot juice, xanthan gum, gellan gum, guar, sodium carboxymethylcellulose were also investigated for viscosity, turbidity and storage time [21]. Hydrocolloids are used in food for their intended purpose and function in water retention, viscosity making, thickeners, volatility, ionization in aqueous solutions, resulting in the ability to stabilize soluble particles in the product, keeping the product stable.

Studies on the effect of each viscous additive have been studied. However, studies on the interaction on the admixture of viscous additives on the physical and chemical properties of turbid fruit juice are limited. Therefore, the objective of this study was to investigate the interaction between xanthan gum and carboxymethylcellulose on the physical and chemical properties of turbid juice from green asparagus. With the surface-response method, the CCD test design will determine the target function model such as viscosity, stability, sensory according to the concentration of xanthan gum and carboxymethylcellulose. At the same time will evaluate if the interaction of xanthan gum and carboxymethylcellulose of meaningful or not to the physio-chemical quality of turbid juice of green asparagus.

\section{EXPERIMENTAL}

The green asparagus (Asparagus officinalis) is purchased from An Phu Dong market, Ho Chi Minh city, Vietnam. Bamboo shoots should be carefully selected to ensure that are not rotten, damaged or bruised. In particular, there must be a fixed source that is not purchased in different places and must be of same type. Bamboo shoots are cleaned and cut to a length of $12 \pm$ $1.5 \mathrm{~cm}$. Viscosity making additives used in this study are xanthan gum purchased from KeKelco Division of Merck and Company Inc. (LT100, Kelco, Co. Ltd, USA) and carboxymethylcellulose (CMC) were purchased at Suzhou Elifa Chemical Co. Ltd. Syrup is used to adjust the brix level of the turbid juice. The syrup is prepared in a ratio of $120 \mathrm{~g}$ of sugar and $80 \mathrm{~g}$ of water, heated at $85^{\circ} \mathrm{C}$ for $20 \mathrm{~min}$.

Preparation of cloudy juice of asparagus: Asparagus was washed after picking up the tops from $12 \pm 1.5 \mathrm{~cm}$ and the fruit juice was prepared according to the flow chart given in Fig. 1.

Experimental design: In this study, the overall effect of xanthan gum and carboxymethylcellulose was investigated. The experiments were performed according to CCD with two independent variables: the concentration of xanthan gum (\%) and the concentration of carboxymethylcellulose (\%), each of which has three levels of values as shown in Table-1. The value intervals of the independent variables were selected based on previous studies. The coding and survey levels used in the experiments are shown in Table-2.

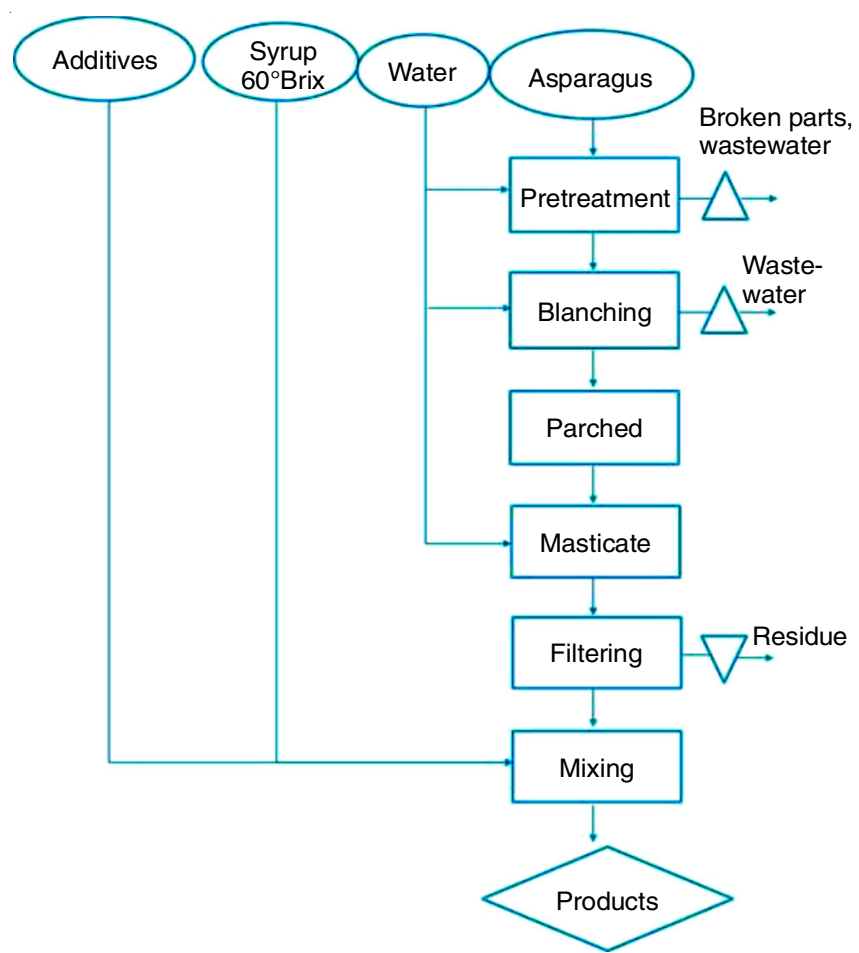

Fig. 1. Green asparagus preparation process

\begin{tabular}{ccc}
\multicolumn{3}{c}{ TABLE-1 } \\
\multicolumn{3}{c}{ EXPERIMENTAL DESIGN } \\
\hline Runs & Xanthan gum (\%) & Carboxymethyl cellulose (\%) \\
\hline 1 & -1 & 1 \\
2 & -1.41421 & 0 \\
3 & 1 & 1 \\
4 & 0 & 0 \\
5 & -1 & -1 \\
6 & 0 & -1.41421 \\
7 & 0 & 0 \\
8 & 0 & 0 \\
9 & 1.41421 & 0 \\
10 & 0 & 1.41421 \\
11 & 0 & 0 \\
12 & 1 & -1 \\
13 & 0 & 0 \\
\hline
\end{tabular}

\begin{tabular}{|c|c|c|c|c|}
\hline \multicolumn{5}{|c|}{$\begin{array}{c}\text { TABLE-2 } \\
\text { VARIABLE ENCODING AND SURVEY LEVE }\end{array}$} \\
\hline \multirow[b]{2}{*}{ Factor } & \multicolumn{3}{|c|}{ Value level } & \multirow{2}{*}{$\begin{array}{l}\text { Variable } \\
\text { range }\end{array}$} \\
\hline & $\begin{array}{c}\text { Above } \\
+1\end{array}$ & $\begin{array}{l}\text { Base level } \\
0\end{array}$ & $\begin{array}{c}\text { Bottom level } \\
-1\end{array}$ & \\
\hline $\mathrm{XG}(\%)$ & 0.15 & 0.10 & 0.05 & 0.05 \\
\hline $\mathrm{CMC}(\%)$ & 0.40 & 0.30 & 0.20 & 0.10 \\
\hline
\end{tabular}

Determination of viscosity: Viscosity measurements were conducted using viscometer (NDJ-5S) as per reported method [20]. The test specimen was stored in a $250 \mathrm{~mL}$ glass jar and thermostatically controlled by a thermostatic tank for about 15-20 min to reach a desired temperature of $30^{\circ} \mathrm{C}$ and then measured for $5 \mathrm{~min}$ at a rotational speed of $60 \mathrm{rpm}$. All samples were measured for the same temperature, time and rotation speed. Each experiment was repeated three times.

Determination of stability: The specimen was centrifuged for $30 \mathrm{~min}$. The sample was measured at $660 \mathrm{~nm}$ before 
and after centrifugation (surface fluid) [19]. The photo lab 7600 UV-VIS device was used to measure the absorption of green asparagus. Each experiment was repeated three times. Stability is derived from relative turbidity ( $\mathrm{T} \%$ ).

$$
\mathrm{T}(\%)=\frac{\mathrm{T}_{\mathrm{c}}}{\mathrm{T}_{\mathrm{o}}} \times 100
$$

where $T_{o}$ and $T_{c}$ are the turbidity before and after the centrifugation, respectively.

Sensory assessment method: Method of assessment according to TCVN 3215-79 preparation follow the steps of sample preparation, coding, instrument preparation, instruction sheets, answer sheets, laboratory temperature and volume of $20 \mathrm{~mL}$ for a sample. The sensory evaluation was conducted at the processing room of Nguyen Tat Thanh University with the participation of 40 peoples, conducted in three rounds of 14 peoples, aging from 20 to 24 years. The tester's answer sheets report is shown in Table-3.

\section{RESULTS AND DISCUSSION}

Effect of xanthan gum and carboxymethylcellulose on viscosity: Fig. 2 shows the surface response of viscosity when changing carboxymethylcellulose (CMC) and xanthan gum concentrations in turbid green asparagus juice. The diagram shows that when increasing the percentage concentration of CMC from 0.2 to $0.4 \%$, the viscosity increases. Similarly, xanthan gum has a concentration of $0.05-0.15 \%$, the viscosity also increases. The simultaneous increase of density for these two substances, the viscosity also increases, typically when $\mathrm{CMC}$ concentration is $0.2 \%$ and xanthan gum concentration is $0.05 \%$, the measured viscosity value is the lowest value of $61.667 \mathrm{mPa} . \mathrm{s}$, but when both were increased to CMC $0.4 \%$, xanthan gum $0.15 \%$, viscosity increased to the highest of 247 $\mathrm{mPa}$.s. The essence of these two substances is the hydrocolloid group in which this group has a hydroxyl group $\left(\mathrm{OH}^{-}\right)$, the presence of this $\mathrm{OH}^{-}$group increases their affinity with the

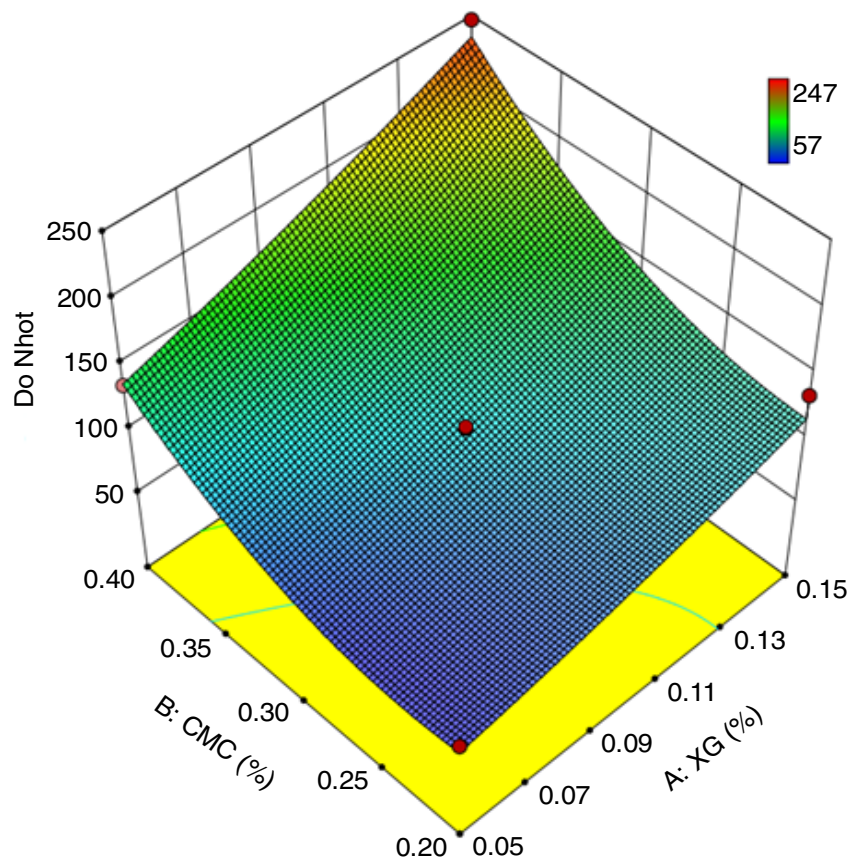

TABLE-3

STATUS DESCRIPTION SAMPLES

\begin{tabular}{ccl}
\hline $\begin{array}{c}\text { Names of } \\
\text { indicators }\end{array}$ & Weights & \multicolumn{1}{c}{ Description } \\
\hline & 5 & Liquid state like filtered water \\
State $(20 \%)$ & 3 & Liquid state like fruit juice \\
& 2 & Glutinous state like syrup \\
& 1 & Casty state like vitamins \\
& 0 & Condensed state like jam \\
& 5 & Dark green state like chili, tomato paste \\
\hline & 4 & Light green \\
Colour $(30 \%)$ & 3 & The green colour is slightly yellowish \\
& 2 & Light yellow \\
& 1 & Dark yellow \\
& 0 & Very dark yellow \\
& 5 & The odour of asparagus is pleasant \\
& 4 & The odour of asparagus is quite strong \\
Odour $(50 \%)$ & 3 & The odour of asparagus is strong \\
& 2 & The odour of asparagus is very strong \\
& 1 & There is no odour of asparagus \\
& 0 & It odours strange \\
\hline
\end{tabular}

water molecule that forms the hydrophilic compound. Hydrocolloids are free-floating so that in the mixture with CMC and xanthan gum they interact and collide with water and hydrophilic constituents, resulting in fluid viscosity [21]. Therefore, the more the CMC and xanthan gum elements are added to the solution, the higher the viscosity in the solution is.

In this study, the model for predicting the change in viscosity of turbid green asparagus juice by CMC and xanthan gum concentrations was also identified and presented as below:

$$
\begin{aligned}
\mu= & 203.8718-116.201 \times \mathrm{XG}-1390.46 \mathrm{C} \times \mathrm{CMC}+ \\
& 1908.333 \times \mathrm{XG} \times \mathrm{CMC}+1657.5 \times \mathrm{XG}^{2}+ \\
& 2831.042 \times \mathrm{CMC}_{2}\left(\mathrm{R}^{2}=0.9732\right)
\end{aligned}
$$

The model has the correlation coefficient of $R^{2}=0.9732$, indicating that the model is highly compatible with experi-

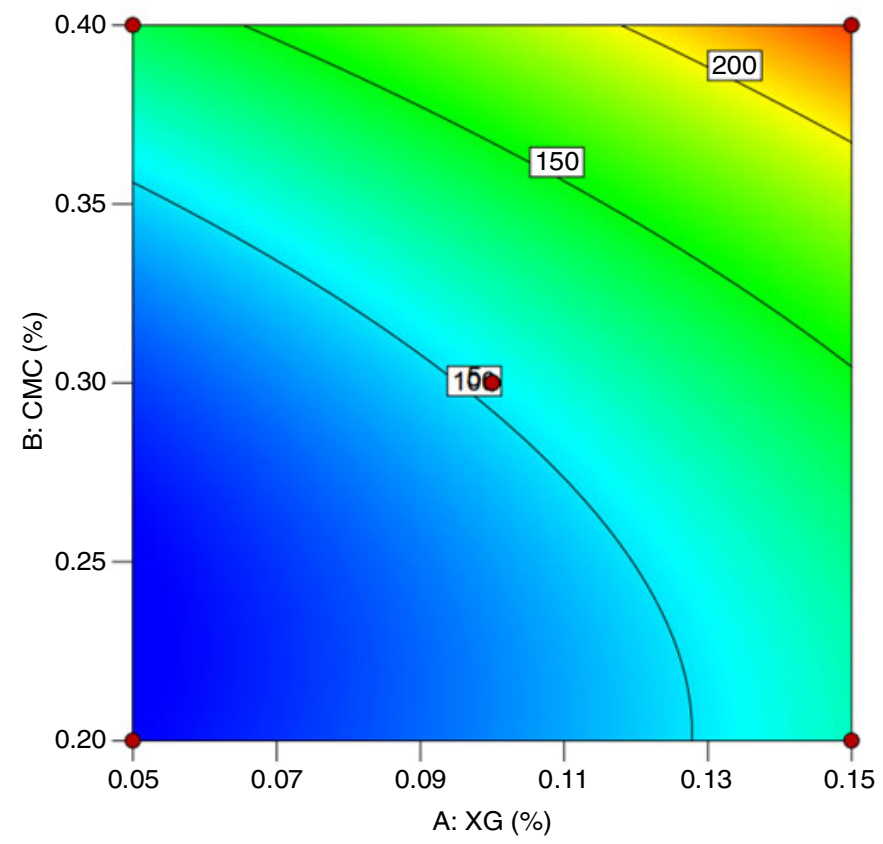

Fig. 2. Effect of xanthan gum $(\mathrm{XG})$ concentrations and $\mathrm{CMC}$ on the viscosity of turbid juice of green asparagus 
mental data. From the results of ANOVA analysis (Table-4), the viscosity is influenced at grade 1 by two factors: xanthan gum and $\mathrm{CMC}$ concentration are affected at grade 2 of CMC concentration $(\mathrm{p}<0.05)$. While the concentration factor level 2 of xanthan gum does not affect significantly $(p>0.05)$ on product viscosity, the interaction of two xanthan gum and CMC factors did not significantly affect the product viscosity ( $\mathrm{p}>$ 0.05). In terms of influencing factors, the concentration CMC level 2 strongly affect the viscosity with positive coefficient 2831.042.

Thus, from the analysis of significant effects of coefficients in the given regression equation, it is concluded that mixing of CMC and xanthan gum was not significant. And the change in $\mathrm{CMC}$ concentration affects the viscosity change of the product more than xanthan gum. Previous study demonstrates that xanthan gum affects the viscosity change greater than that of CMC due to xanthan gum has a molecular structure larger than CMC, so hydration is higher than CMC. However, in this study, the result was a change in the mixing of xanthan gum with CMC. The impact of CMC is higher. When mixing two viscous additives, the interaction can occur resonant or antagonistic. In this case, the results show that the mixing reduces the viscosity of xanthan gum but increases the viscosity of $\mathrm{CMC}$ in the turbid juice from green asparagus.

Impact of xanthan gum and carboxymethylcellulose on stability: Fig. 3 shows the interaction of xanthan gum and $\mathrm{CMC}$ on fruit juice stability. When the CMC concentration increases from 0.2 to $0.4 \%$, the stability increases, and xanthan gum increases from 0.05 to $0.15 \%$. The fruit juice stability also increased which the lowest and highest increase was 4,922 and $81.5 \%$, respectively. Static propulsion is a stable component for particles in the dispersion system. Fruit juice particles have a negative charge, so when added to hydrocolloids, they also have negative charges, as two charges with the same sign give rise to high repulsion and even dispersion in fruit juices, so stability increased [18].

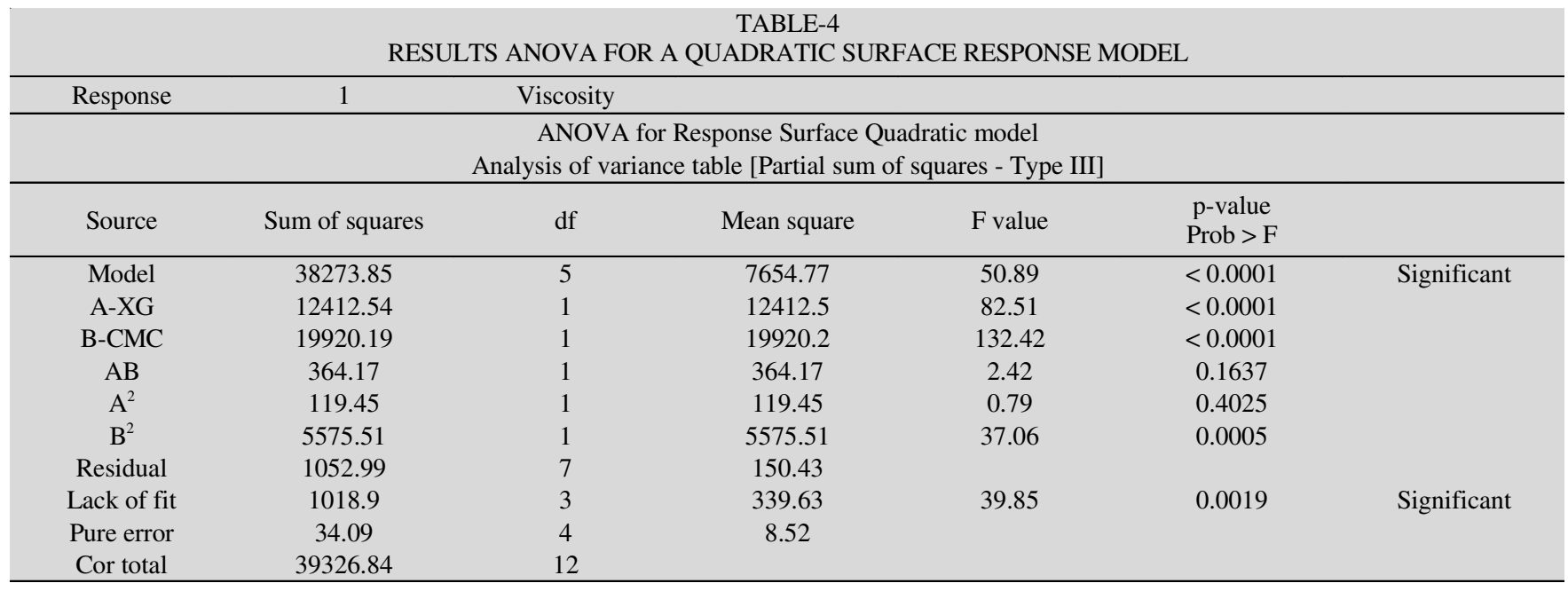
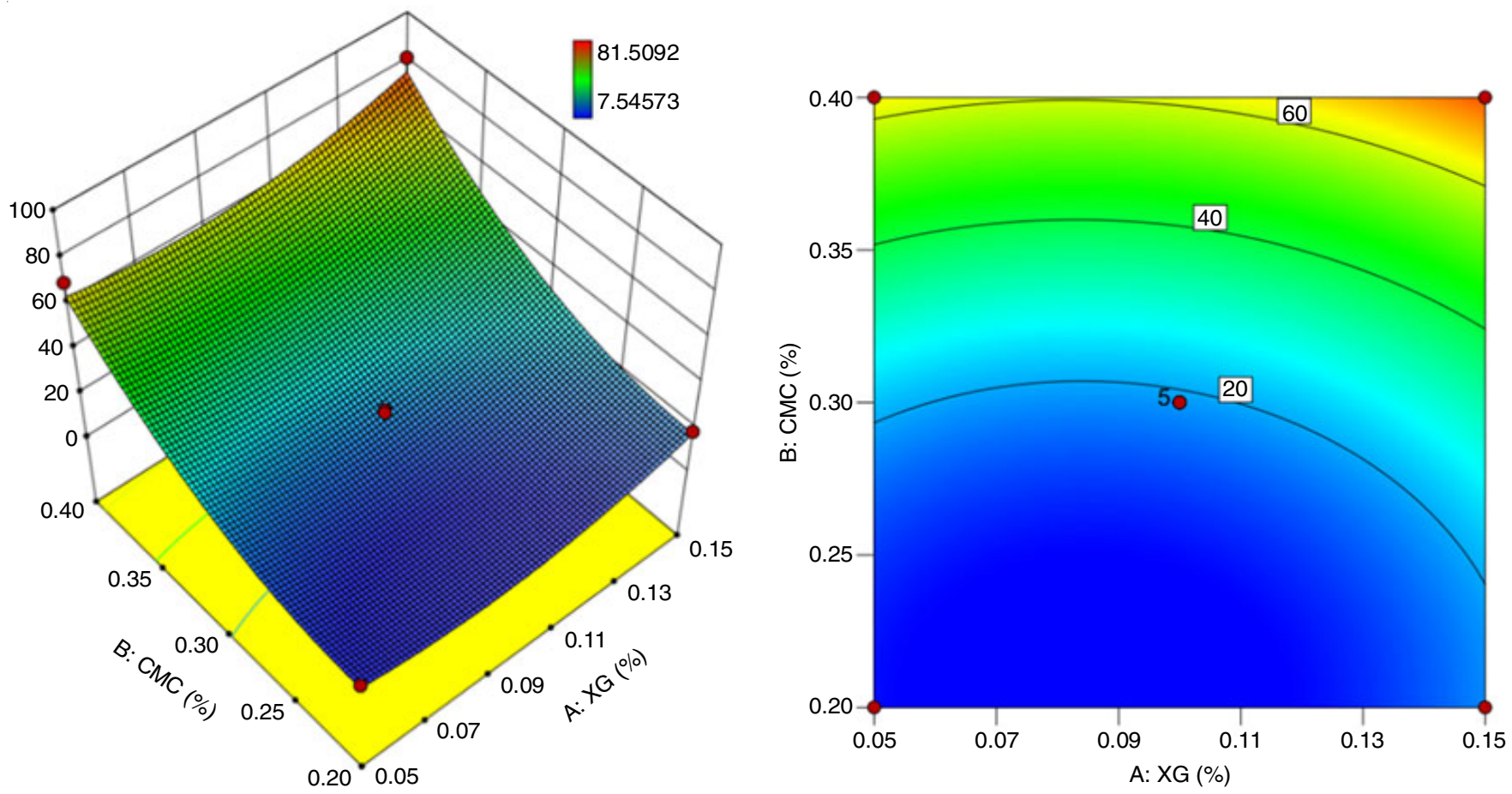

Fig. 3. Effect of xanthan gum $(\mathrm{XG})$ concentrations and $\mathrm{CMC}$ on the stability of turbid asparagus juice 
The following regression equation describes the effect of xanthan gum and CMC concentrations on the stability of turbid juice from green asparagus:

$$
\begin{aligned}
& \mathrm{T}(\%)=89.35135-590.565 \times \mathrm{XG}-592.002 \times \\
& \mathrm{CMC}+126.806 \times \mathrm{XG} \times \mathrm{CMC}+3282.184 \times \\
& \mathrm{XG}^{2}+1438.341 \times \mathrm{CMC}^{2} \quad\left(\mathrm{R}^{2}=0.9813\right)
\end{aligned}
$$

The model has a correlation coefficient $R^{2}=0.9813$, indicating that the model is highly compatible with experimental data. From the analysis of ANOVA (Table-5), the stability is influenced by grade 1 of a factor, namely CMC and grade of factor 1 , namely xanthan gum and is influenced by grade 2 of concentration $\mathrm{CMC}$ and xanthan gum $(\mathrm{p}<0.05)$. In addition, the interaction between the two factors xanthan gum and CMC also did not affect the stability of the product ( $p>0.05$ ). From the analyses of the factors influencing the stability, it was found that pre-xanthan gum coefficient was positive of 3282,184 and 2.2 times higher than that of the positive of 1438,341 of CMC factor grade 2 . The degree of stability of green asparagus juice is more influenced by xanthan gum than CMC.

Thus, from the analysis of significant effects of coefficients in the given regression equation, it was concluded that when mixing CMC and xanthan gum, coefficient of interaction of xanthan gum and $\mathrm{CMC}$ was not significant $(\mathrm{p}>0.05)$. The coefficient before the grade 1 interaction factor of $\mathrm{CMC}$ and grade
1 of xanthan gum with the coefficient before grade 2 interaction factor of CMC and xanthan gum are also significant $(\mathrm{p}<0.05)$.

Effect of xanthan gum and carboxymethylcellulose on sensory quality: The sensory results according to TCVN 321579 with the score of 6 are shown in Table-6. The sensory evaluation results were based on three criteria: status, colour, and odour of turbid juice of green asparagus. At $5 \%$ significance level, the results show a significant differences between fresh samples and all other mixed CMC and xanthan gum samples (Table-6). Meanwhile, when fixing the concentration of xanthan gum at $0.05 \%$ and increasing the CMC concentration from $0.2-0.4 \%$, there is a considerable difference in state between sample 2 and 3 . If fixed both concentration CMC level at $0.3 \%$ and $\mathrm{XG}$ concentration at $0.1 \%$, there is no significant difference compared to the remaining samples. On the other hand, I changing CMC concentrations from 0.2 to $0.4 \%$ and fixed xanthan gum at $0.15 \%$, then samples 12 and 13 were also differed in status but were not significant. There is a similar difference to the state but only the difference between the fresh sample and all the other samples without significant differences between the samples. The changes in xanthan gum and CMC concentrations have only a significant effect on the status indicator of the samples, with no significant difference in colour and odour [18]. For the production technology of turbid fruit juices, this result is a good development direction

TABLE-5

ANOVA RESULT FOR THE QUADRATIC SURFACE MODEL RESPONDING TO THE OBJECTIVE FUNCTION IS THE STABILITY

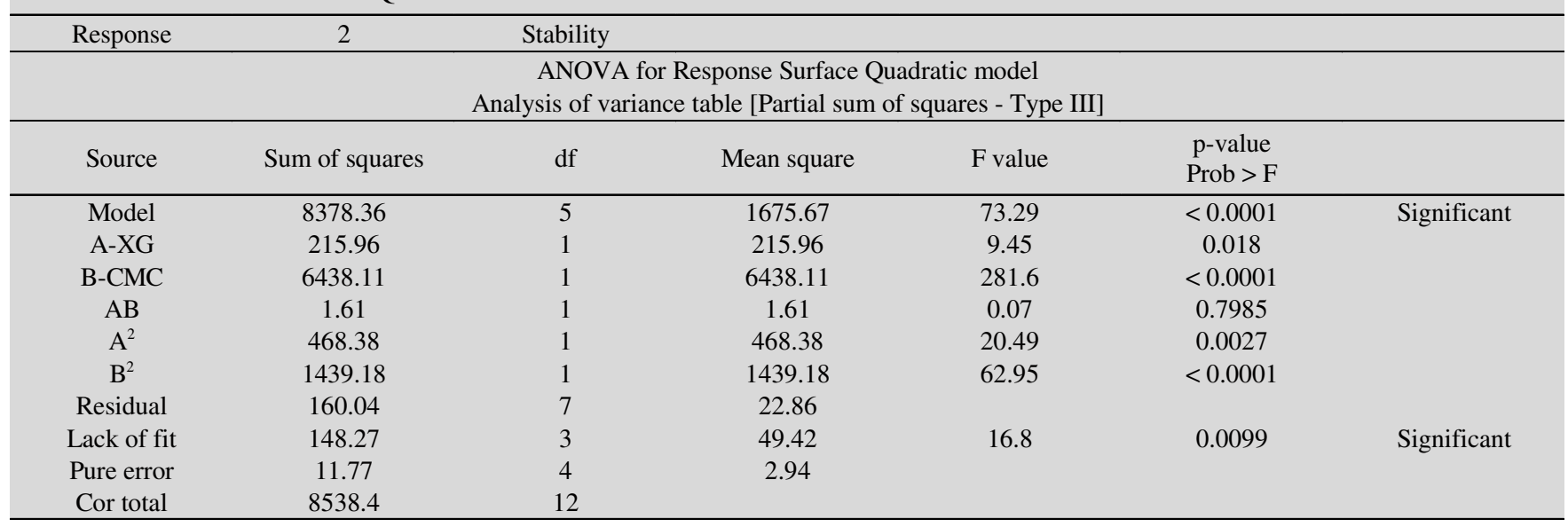

TABLE-6

EFFECTS OF XG AND CMC CONCENTRATIONS ON THE SENSORY OF TURBID ASPARAGUS JUICE

\begin{tabular}{cccccc}
\hline No. & XG concentration $(\%)$ & CMC concentration $(\%)$ & State & Colour & Odour \\
\hline 0 & 0 & 0 & $4.90 \pm 0.44^{\mathrm{a}}$ & $4.95 \pm 0.22^{\mathrm{a}}$ & $4.93 \pm 0.27^{\mathrm{a}}$ \\
1 & 0.029289 & 0.3 & $3.40 \pm 0.49^{\text {cde }}$ & $3.9 \pm 0.3^{\mathrm{b}}$ & $3.9 \pm 0.3^{\mathrm{b}}$ \\
2 & 0.05 & 0.2 & $3.30 \pm 0.46^{\text {de }}$ & $3.9 \pm 0.3^{\mathrm{b}}$ & $3.85 \pm 0.36^{\mathrm{b}}$ \\
3 & 0.05 & 0.4 & $3.90 \pm 0.37^{\mathrm{b}}$ & $3.97 \pm 0.15^{\mathrm{b}}$ & $3.9 \pm 0.3^{\mathrm{b}}$ \\
4 & 0.1 & 0.158579 & $3.35 \pm 0.48^{\text {de }}$ & $3.9 \pm 0.3^{\mathrm{b}}$ & $3.87 \pm 0.33^{\mathrm{b}}$ \\
5 & 0.1 & 0.3 & $3.50 \pm 0.50^{\text {cd }}$ & $3.95 \pm 0.22^{\mathrm{b}}$ & $3.83 \pm 0.38^{\mathrm{b}}$ \\
6 & 0.1 & 0.3 & $3.58 \pm 0.54^{\mathrm{c}}$ & $3.93 \pm 0.26^{\mathrm{b}}$ & $3.88 \pm 0.33^{\mathrm{b}}$ \\
7 & 0.1 & 0.3 & $3.28 \pm 0.45^{\text {ef }}$ & $3.95 \pm 0.22^{\mathrm{b}}$ & $3.82 \pm 0.38^{\mathrm{b}}$ \\
8 & 0.1 & 0.3 & $3.43 \pm 0.5^{\text {cde }}$ & $3.98 \pm 0.15^{\mathrm{b}}$ & $3.92 \pm 0.27^{\mathrm{b}}$ \\
9 & 0.1 & 0.3 & $3.32 \pm 0.47^{\text {de }}$ & $3.95 \pm 0.22^{\mathrm{b}}$ & $3.85 \pm 0.36^{\mathrm{b}}$ \\
10 & 0.1 & 0.441421 & $3.37 \pm 0.49^{\text {cdef }}$ & $3.98 \pm 0.15^{\mathrm{b}}$ & $3.9 \pm 0.3^{\mathrm{b}}$ \\
12 & 0.15 & 0.2 & $3.80 \pm 0.46^{\mathrm{b}}$ & $3.95 \pm 0.22^{\mathrm{b}}$ & $3.87 \pm 0.33^{\mathrm{b}}$ \\
13 & 0.15 & 0.4 & $3.38 \pm 0.49^{\text {cdef }}$ & $3.88 \pm 0.33^{\mathrm{b}}$ & $3.85 \pm 0.36^{\mathrm{b}}$ \\
\hline
\end{tabular}


for the juice industry, especially for turbid fruit juice from asparagus [20].

\section{Conclusion}

The present study evaluated the influence of xanthan gum and carboxymethylcellulose (CMC) concentrations on quality indicators, namely state, odour and colour, of the turbid fruit juice from asparagus. It was found that the simultaneous addition of $\mathrm{CMC}$ and xanthan gum was not significant ( $\mathrm{p}>0.05)$. However, when the CMC concentration increased, the viscosity increased which was a significant difference. The increase in xanthan gum concentration also increases the product stability with significant difference $(\mathrm{p}<0.05)$. Finally, CMC and xanthan gum have no significant effect on the colour and odour of the product. This result suggests a new way to improve the consumer acceptability to the turbid juice by enhancing the stability of turbid juice of green asparagus juice without compromising odour and colour characteristics.

\section{CONFLICT OF INTEREST}

The authors declare that there is no conflict of interests regarding the publication of this article.

\section{REFERENCES}

1. T.S. Vo, D.H. Ngo, L.G. Bach, D.N. Ngo and S.K. Kim, Process Biochem., 54, 188 (2017); https://doi.org/10.1016/j.procbio.2017.01.001.

2. N.P.T. Nhan, T.T. Hien, L.T.H. Nhan, P.N.Q. Anh, L.T. Huy, T.C.T. Nguyen, D.T. Nguyen and L.G. Bach, Diffus. Defect Data Solid State Data Pt. B Solid State Phenom., 279, 235 (2018);

https://doi.org/10.4028/www.scientific.net/SSP.279.235.

3. A.N.Q. Phan, L.G. Bach, T.D. Nguyen and N.T.H. Le, J. Nanosci. Nanotechnol., 19, 974 (2019); https://doi.org/10.1166/jnn.2019.15925.

4. T.T. Hien, N.P.T. Nhan, N.D. Trinh, V.T.T. Ho and L.G. Bach, Diffus. Defect Data Solid State Data Pt. B Solid State Phenom., 279, 217 (2018);

https://doi.org/10.4028/www.scientific.net/SSP.279.217.
5. T. Tran, H. Nguyen, D. Nguyen, T. Nguyen, H. Tan, L. Nhan, D. Nguyen, L. Tran, S. Do and T. Nguyen, Processes, 6, 206 (2018); https://doi.org/10.3390/pr6110206.

6. N. Shiomi, Phytochemistry, 20, 2581 (1981); https://doi.org/10.1016/0031-9422(81)83099-3.

7. J. Wang, Y. Liu, J. Zhao, W. Zhang and X. Pang, J. Sci. Food Agric., 93, 1492 (2013); https://doi.org/10.1002/jsfa.5922.

8. S. Bousserouel, J. Le Grandois, F. Gossé, D. Werner, S.W. Barth, E. Marchioni, J. Marescaux and F. Raul, Int. J. Oncol., 43, 394 (2013); https://doi.org/10.3892/ijo.2013.1976.

9. S. Jaramillo, F.J.G. Muriana, R. Guillen, A. Jimenez-Araujo, R. Rodriguez-Arcos and S. Lopez, J. Funct. Foods, 26, 1 (2016); https://doi.org/10.1016/j.jff.2016.07.007.

10. J. Zhao, W. Zhang, X. Zhu, D. Zhao, K. Wang, R. Wang and W. Qu, J. Sci. Food Agric., 91, 2095 (2011); https://doi.org/10.1002/jsfa.4429.

11. Y.G. Ku, D.H. Kang, C.K. Lee, S.Y. Lee, C.S. Ryu, D.E. Kim, M. Polovka, J. Namieœnik and S. Gorinstein, Food Chem., 244, 349 (2018); https://doi.org/10.1016/j.foodchem.2017.10.044.

12. R.E. Lill, New Zealand J. Exp. Agric., 8, 163 (1980); https://doi.org/10.1080/03015521.1980.10426252.

13. G.S. Johannessen, S. Loncarevic and H. Kruse, Int. J. Food Microbiol., 77, 199 (2002); https://doi.org/10.1016/S0168-1605(02)00051-X.

14. F. Tezcan, M. Gültekin-Özgüven, T. Diken, B. Özçelik and F.B. Erim, Food Chem., 115, 873 (2009); https://doi.org/10.1016/j.foodchem.2008.12.103.

15. National Research Council (U.S.), National Academies Press: Washington, D.C. (2007).

16. T. Beveridge, Crit. Rev. Food Sci. Nutr., 42, 317 (2002); https://doi.org/10.1080/10408690290825556.

17. E.M. Coelho, R.G. Gomes, B.A.S. Machado, R.S. Oliveira, M.S. Lima, L.C. de Azêvedo and M.A.U. Guez, Food Hydrocoll., 62, 158 (2017); https://doi.org/10.1016/j.foodhyd.2016.07.027.

18. D.B. Genovese and J.E. Lozano, Food Hydrocoll., 15, 1 (2001); https://doi.org/10.1016/S0268-005X(00)00053-9.

19. C. Liang, X. Hu, Y. Ni, J. Wu, F. Chen and X. Liao, Food Hydrocoll., 20, 1190 (2006); https://doi.org/10.1016/j.foodhyd.2006.01.010.

20. G.E. Ibrahim, I.M. Hassan, A.M. Abd-Elrashid, K.F. El-Massry, A.H. Eh-Ghorab, M.R. Manal and F. Osman, Food Hydrocoll., 25, 91 (2011); https://doi.org/10.1016/j.foodhyd.2010.05.009.

21. D. Saha and S. Bhattacharya, J. Food Sci. Technol., 47, 587 (2010); https://doi.org/10.1007/s13197-010-0162-6. 